\title{
Fasting before blood lipid testing might be unnecessary
}

A new study indicates that measuring the level of LDL cholesterol in the blood of a patient who has not previously fasted has the same prognostic value as measuring it after fasting, as currently recommended in clinical guidelines. Fasting for 8-12 h before phlebotomy can be inconvenient for patients, and the new finding indicates that fasting might not provide a morerepresentative measure of particular lipid parameters (such as triglyceride level), as was previously thought.

The investigators used data from NHANES-III, which is a representative database of the US population. A total of 16,161 patients ( $62 \%$ fasting, $38 \%$ nonfasting) were suitable for inclusion in the analysis, 4,299 of whom from each group were included after propensity score matching. In this matched cohort, the fasting and nonfasting LDL-cholesterol levels provided similar prognostic value (C-statistic 0.59, 95\% CI 0.57-0.61 vs C-statistic 0.58, 95\% CI 0.56-0.60;
$P=0.73$ ) for the primary outcome of allcause mortality. Likewise, for the secondary outcome of cardiovascular mortality, the prognostic value of the fasting LDL-cholesterol level (C-statistic 0.62, 95\% CI 0.60-0.66) was similar to that of the nonfasting LDL-cholesterol level (C-statistic 0.62, 95\% CI 0.60-0.66; $P=0.96)$. Similar results were obtained for triglyceride and total-cholesterol levels.

The researchers believe that the similarity in prognostic value of fasting and nonfasting lipid levels means that "national and international agencies should consider re-evaluating the recommendation that patients fast before [clinicians obtain] a lipid panel".

Gregory B. Lim

Original article Doran, B. et al. Prognostic value of fasting vs. non-fasting low density lipoprotein cholesterol levels on long-term mortality: insight from the National Health and Nutrition Survey III (NHANES-III). Circulation doi:10.1161/ CIRCULATIONAHA.114.010001 